\title{
Syntheses of Ficuseptine, Juliprosine, and Juliprosopine by Biomimetic Intramolecular Chichibabin Pyridine Syntheses
}

\author{
Barry B. Snider* and Bobbianna J. Neubert \\ Department of Chemistry MS 015, Brandeis University, Waltham, MA 02454-9110 \\ snider@brandeis.edu
}

Supporting Material

$\begin{array}{ll}\text { Experimental Procedures } & \text { S2 }\end{array}$

Copies of ${ }^{1} \mathrm{H}$ and ${ }^{13} \mathrm{C}$ NMR spectra $\quad$ S11-S46 
General. NMR spectra were recorded at $400 \mathrm{MHz}$. Chemical shifts are reported in $\delta$ and coupling constants in $\mathrm{Hz}$. IR spectra are reported in $\mathrm{cm}^{-1}$. Filtration through ion exchange resin was carried out with Dowex 1X8-400 (strongly basic anion exchange in the chloride form) using $\mathrm{MeOH}$ as the eluent.

6,8-Diheptyl-2,3-dihydro-1H-indolizinium Chloride (3a). Nonanal (4a) (1.2 mL, 6.7 mmol) was added to a solution of 4-aminobutyraldehyde dimethyl acetal $(6)(0.45 \mathrm{~mL}, 3.3$ mmol) in $33 \mathrm{~mL}$ of acetic acid. The clear solution was stirred at $95{ }^{\circ} \mathrm{C}$ for $2 \mathrm{~d}$ giving a dark solution. The mixture was cooled to $25{ }^{\circ} \mathrm{C}$ and basified with $1 \mathrm{M} \mathrm{NaOH}$. The aqueous layer was washed with hexane $(2 \times 50 \mathrm{~mL})$, saturated with $\mathrm{NaCl}$, and extracted with $\mathrm{CHCl}_{3}(3 \times 75 \mathrm{~mL})$. The combined $\mathrm{CHCl}_{3}$ layers were dried over $\mathrm{CaCl}_{2}$ and concentrated to provide a dark oil. Flash chromatography on silica gel $\left(9: 1 \mathrm{CHCl}_{3} / \mathrm{MeOH}\right)$ followed by filtration through ion exchange resin yielded $0.78 \mathrm{~g}(66 \%)$ of pure $3 \mathrm{a}$ as a dark oil: ${ }^{1} \mathrm{H}$ NMR $\left(\mathrm{CD}_{3} \mathrm{OD}\right) 8.66(\mathrm{~s}, 1), 8.18(\mathrm{~s}, 1)$, $4.88(\mathrm{t}, 2, J=7.5), 3.49(\mathrm{t}, 2, J=7.5), 2.82(\mathrm{t}, 4, J=7.5), 2.52(\mathrm{tt}, 2, J=7.5,7.5), 1.70-1.63(\mathrm{~m}$, 4), 1.39-1.29 (m, 16), $0.92(\mathrm{t}, 3, J=7.3), 0.86(\mathrm{t}, 3, J=7.3) ;{ }^{1} \mathrm{H} \mathrm{NMR}\left(\mathrm{CDCl}_{3}\right) 9.36(\mathrm{~s}, 1), 7.82$ $(\mathrm{s}, 1), 5.32(\mathrm{t}, 2, J=7.9), 3.43(\mathrm{t}, 2, J=7.9), 2.81(\mathrm{t}, 2, J=7.9), 2.72(\mathrm{t}, 2, J=7.9), 2.59(\mathrm{tt}, 2, J$ $=7.9,7.9), 1.70-1.60(\mathrm{~m}, 4), 1.36-1.28(\mathrm{~m}, 16), 0.89(\mathrm{t}, 3, J=6.7), 0.88(\mathrm{t}, 3, J=6.7) ;{ }^{13} \mathrm{C}$ NMR $\left(\mathrm{CD}_{3} \mathrm{OD}\right) 156.7,146.0,142.9,140.8,139.0,60.7,33.3,33.04,33.03,32.9,31.9,31.8,30.40$, 30.37, 30.27 (2 C), 30.2, 23.8 (2 C), 22.2, 14.6 (2 C); IR (neat) 3406, 1627, 1506, 1466; HRMS (DEI) Calcd for $\mathrm{C}_{22} \mathrm{H}_{38} \mathrm{~N}\left(\mathrm{M}^{+}\right)$316.3004, found 316.2994.

Nonanal (4a) (0.5 mL, $2.9 \mathrm{mmol})$ was added to a solution of 1-pyrroline ${ }^{7}$ (5) (100 mg, 1.4 $\mathrm{mmol}$ ) in $14 \mathrm{~mL}$ of acetic acid. The clear solution was stirred at $25^{\circ} \mathrm{C}$ for $24 \mathrm{~h}$ giving a yellow solution. The mixture was basified with $1 \mathrm{M} \mathrm{NaOH}$, washed with hexane $(2 \times 15 \mathrm{~mL})$, saturated with $\mathrm{NaCl}$, and extracted with $\mathrm{CHCl}_{3}(3 \times 20 \mathrm{~mL})$. The combined $\mathrm{CHCl}_{3}$ layers were dried over $\mathrm{CaCl}_{2}$ and concentrated to provide an orange oil. Flash chromatography on silica gel $(9: 1$ $\left.\mathrm{CHCl}_{3} / \mathrm{MeOH}\right)$ followed by filtration through ion exchange resin yielded $131 \mathrm{mg}(52 \%)$ of pure 3a as a yellow oil. 
2,3-Dihydro-6,8-diphenyl-1H-indolizinium Chloride (3b). Phenylacetaldehyde (4b) (0.16 g, $1.3 \mathrm{mmol})$ was added to a solution of 4-aminobutyraldehyde dimethyl acetal (6) $(93 \mu \mathrm{L}, 0.66$ $\mathrm{mmol}$ ) in $7 \mathrm{~mL}$ of acetic acid. The clear solution was stirred at $95^{\circ} \mathrm{C}$ for $2 \mathrm{~d}$ giving a dark solution. The mixture was cooled to $25^{\circ} \mathrm{C}$ and basified with $1 \mathrm{M} \mathrm{NaOH}$. The aqueous layer was washed with $\mathrm{Et}_{2} \mathrm{O}(2 \times 75 \mathrm{~mL})$, saturated with $\mathrm{NaCl}$, and extracted with $\mathrm{CHCl}_{3}(3 \times 100 \mathrm{~mL})$. The combined $\mathrm{CHCl}_{3}$ layers were dried over $\mathrm{CaCl}_{2}$ and concentrated to provide an orange oil. Flash chromatography on silica gel $\left(4: 1 \mathrm{CHCl}_{3} / \mathrm{MeOH}\right)$ followed by filtration through ion exchange resin yielded $120 \mathrm{mg}(58 \%)$ of pure $3 \mathbf{b}$ as a pale yellow solid: $\mathrm{mp} 264-266{ }^{\circ} \mathrm{C} ;{ }^{1} \mathrm{H}$ $\operatorname{NMR}\left(\mathrm{CDCl}_{3}\right) 10.24(\mathrm{~s}, 1), 8.29(\mathrm{~s}, 1), 7.88(\mathrm{~d}, 2, J=7.3), 7.59-7.46(\mathrm{~m}, 8), 5.57(\mathrm{t}, 2, J=7.3)$, $3.60(\mathrm{t}, 2, J=7.3), 2.58(\mathrm{tt}, 2, J=7.3,7.3) ;{ }^{13} \mathrm{C} \mathrm{NMR}\left(\mathrm{CDCl}_{3}\right)$ 154.0, 141.4, 139.6, 139.4, 138.4, 134.3, 132.8, 130.2, 130.0, 129.7 (2 C), 129.4 (2 C), 128.4 (2 C), 127.6 (2 C), 60.5, 32.4, 22.3; IR (KBr) 3406, 1621, 1484; HRMS (FAB, $\mathrm{CH}_{2} \mathrm{Cl}_{2} / \mathrm{NBA}$ ) Calcd for $\mathrm{C}_{20} \mathrm{H}_{18} \mathrm{~N}\left(\mathrm{M}^{+}\right)$272.1439, found 272.1429 .

Phenylacetaldehyde (4b) (0.26 g, $1.1 \mathrm{mmol})$ was added to a solution of 1-pyrroline ${ }^{7}$ (5) (75 $\mathrm{mg}, 2.2 \mathrm{mmol}$ ) in $11 \mathrm{~mL}$ of acetic acid. The clear solution was stirred at $25^{\circ} \mathrm{C}$ for $24 \mathrm{~h}$ giving a yellow solution. The mixture was basified with $1 \mathrm{M} \mathrm{NaOH}$, washed with $\mathrm{Et}_{2} \mathrm{O}(2 \times 30 \mathrm{~mL})$, saturated with $\mathrm{NaCl}$, and extracted with $\mathrm{CHCl}_{3}(3 \times 50 \mathrm{~mL})$. The combined $\mathrm{CHCl}_{3}$ layers were dried over $\mathrm{CaCl}_{2}$ and concentrated to provide an orange oil. Flash chromatography on silica gel (4:1 $\left.\mathrm{CHCl}_{3} / \mathrm{MeOH}\right)$ followed by filtration through ion exchange resin yielded $175 \mathrm{mg}(68 \%)$ of pure $3 \mathbf{b}$ as a pale yellow solid.

\section{2,3-Dihydro-6,8-bis(4-methoxyphenyl)-1H-indolizinium Chloride (Ficuseptine, 1).}

4-Methoxyphenylacetaldehyde (4c) (0.49 g, $3.4 \mathrm{mmol})$ was added to a solution of 4-aminobutyraldehyde dimethyl acetal $(6)(0.23 \mathrm{~mL}, 1.6 \mathrm{mmol})$ in $16 \mathrm{~mL}$ of acetic acid. The clear solution was stirred at $95{ }^{\circ} \mathrm{C}$ for $2 \mathrm{~d}$ giving a dark solution. The mixture was cooled to $25^{\circ} \mathrm{C}$ and basified with $1 \mathrm{M} \mathrm{NaOH}$. The aqueous layer was washed with $\mathrm{Et}_{2} \mathrm{O}(2 \times 100 \mathrm{~mL})$, saturated with $\mathrm{NaCl}$, and extracted with $\mathrm{CHCl}_{3}(3 \times 150 \mathrm{~mL})$. The combined $\mathrm{CHCl}_{3}$ layers were dried over $\mathrm{CaCl}_{2}$ and concentrated to provide an orange oil. Flash chromatography on silica gel $\left(17: 3 \mathrm{CHCl}_{3} / \mathrm{MeOH}\right)$ 
followed by filtration through ion exchange resin yielded $0.31 \mathrm{~g}(52 \%)$ of pure 1 as a pale yellow solid: $\operatorname{mp~} 185-187^{\circ} \mathrm{C} ;{ }^{1} \mathrm{H}$ NMR $\left(\mathrm{CDCl}_{3}\right) 10.12$ (s, 1), $8.14(\mathrm{~s}, 1), 7.79(\mathrm{~d}, 2, J=8.5), 7.51$ (d, 2, $J=8.5), 7.07(\mathrm{~d}, 2, J=8.5), 6.92(\mathrm{~d}, 2, J=8.5), 5.45(\mathrm{t}, 2, J=7.3), 3.88(\mathrm{~s}, 3), 3.75(\mathrm{~s}, 3), 3.62$ $(\mathrm{t}, 2, J=7.3), 2.54(\mathrm{tt}, 2, J=7.3,7.3) ;{ }^{13} \mathrm{C} \mathrm{NMR}\left(\mathrm{CDCl}_{3}\right) 160.9,160.6,152.9,139.8,138.4$, 137.8, 137.7, 129.8 (2 C), 128.7 (2 C), 126.4, 124.9, 114.8 (2 C), 114.6 (2 C), 60.1, 55.4, 55.2, 32.3, 22.2; IR (KBr) 1607, 1520, 1490; HRMS (FAB, $\left.\mathrm{CH}_{2} \mathrm{Cl}_{2} / \mathrm{NBA}\right)$ Calcd for $\mathrm{C}_{22} \mathrm{H}_{22} \mathrm{NO}_{2}\left(\mathrm{M}^{+}\right)$ 332.1651, found 332.1637. The spectral data are identical to those previously reported. ${ }^{1,5}$

2-Methyl-6-[12-[(tetrahydro-2H-pyran-2-yl)oxy]dodecyl]-3-[[2-(trimethylsilyl)ethoxy]methoxy]-pyridine (11). A solution of 2-[(12-bromododecyl)oxy]tetrahydro-2H-pyran (10) ${ }^{15}$ (6.7 $\mathrm{g}, 19.2 \mathrm{mmol})$ in $9.1 \mathrm{~mL}$ of THF was slowly added to a flask containing Mg turnings (0.56 g, $23.0 \mathrm{mmol}$ ). The solution was heated at $50^{\circ} \mathrm{C}$ for $3 \mathrm{~h}$ to form the Grignard reagent. ${ }^{15 \mathrm{~b}}$ This warm solution was added by cannula to a solution of 6-bromo-2-methyl-3-[[2(trimethylsilyl)ethoxy]methoxy]-pyridine $(\mathbf{9})^{13}(3.05 \mathrm{~g}, 9.6 \mathrm{mmol})$ and $\mathrm{Ni}(\mathrm{dppp}) \mathrm{Cl}_{2}([1,3-$ bis(diphenylphospino)propane]nickel dichloride) $(0.14 \mathrm{~g}, 0.26 \mathrm{mmol})$ in $6 \mathrm{~mL}$ of THF. The dark solution was stirred for $18 \mathrm{~h}$ and treated with saturated aqueous $\mathrm{NH}_{4} \mathrm{Cl}(20 \mathrm{~mL})$. The organic phase was separated and the aqueous phase was extracted with benzene $(3 \times 20 \mathrm{~mL})$. The combined organic layers were washed with $2 \mathrm{M} \mathrm{K}_{2} \mathrm{CO}_{3}(60 \mathrm{~mL})$, dried over $\mathrm{Na}_{2} \mathrm{SO}_{4}$ and concentrated to give an orange oil. Flash chromatography on silica gel (9:1 hexanes/EtOAc) yielded $3.04 \mathrm{~g}(62 \%)$ of 11 as a clear oil: ${ }^{1} \mathrm{H}$ NMR $\left(\mathrm{CDCl}_{3}\right) 7.24(\mathrm{~d}, 1, J=8.2), 6.87(\mathrm{~d}, 1, J=$ 8.2), $5.18(\mathrm{~s}, 2), 4.55-4.54(\mathrm{~m}, 1), 3.87-3.81(\mathrm{~m}, 1), 3.72(\mathrm{t}, 2, J=8.5), 3.73-3.67(\mathrm{~m}, 1), 3.49-$ $3.44(\mathrm{~m}, 1), 3.38-3.32(\mathrm{~m}, 1), 2.65(\mathrm{t}, 2, J=7.9), 2.43(\mathrm{~s}, 3), 1.87-1.42(\mathrm{~m}, 8), 1.36-1.23(\mathrm{~m}, 18)$, $0.92(\mathrm{t}, 2, J=8.5),-0.03(\mathrm{~s}, 9) ;{ }^{13} \mathrm{C}$ NMR $\left(\mathrm{CDCl}_{3}\right)$ 154.2, 149.6, 148.1, 121.2, 120.1, 98.7, 92.9, 67.6, 66.2, 62.2, 37.6, 30.7, 30.4, 29.7, 29.5-29.4 (7 C), 26.2, 25.4, 19.6, 19.4, 18.0, -1.5 (3 C); IR (neat) 2925, 2853, 1465.

(2 $\alpha, 3 \alpha, 6 \alpha)-2-M e t h y l-3-[[2-(t r i m e t h y l s i l y l) e t h o x y] m e t h o x y]-6-[12-[(t e t r a h y d r o-2 H-$ pyran-2-yl)oxy]dodecyl]-piperidine (12). $5 \% \mathrm{Rh} / \mathrm{Al}_{2} \mathrm{O}_{3}(1.8 \mathrm{~g})$ was added to a solution of 11 (3.0 g, $5.9 \mathrm{mmol}$ ) in $\mathrm{CH}_{3} \mathrm{OH}(49 \mathrm{~mL})$. The solution was shaken under $50 \mathrm{psi}$ of $\mathrm{H}_{2}$ for $24 \mathrm{~h}$, 
filtered through Celite, and concentrated to give $2.7 \mathrm{~g}(90 \%)$ of crude 12 as a clear oil that was used without purification: ${ }^{1} \mathrm{H}$ NMR $\left(\mathrm{CDCl}_{3}\right) 4.75(\mathrm{~d}, 1, J=7.0), 4.65(\mathrm{~d}, 1, J=7.0), 4.54-4.50$ $(\mathrm{m}, 1), 3.84-3.79(\mathrm{~m}, 1), 3.70-3.56(\mathrm{~m}, 4), 3.46-3.43(\mathrm{~m}, 1), 3.35-3.30(\mathrm{~m}, 1), 3.13-3.12(\mathrm{~m}, 1)$, 2.94-2.86 (m, 1), 2.08-1.18 (m, 36), $0.85(\mathrm{t}, 2, J=8.5),-0.04(\mathrm{~s}, 9) ;{ }^{13} \mathrm{C} \mathrm{NMR}\left(\mathrm{CDCl}_{3}\right) 98.6$, 93.0, 70.3, 67.5, 65.3, 62.1, 57.5, 55.9, 33.5 (br), 30.6, 29.6, 29.5-29.3 (7 C), 27.2, 26.1, 25.5, 25.3, 22.7 (br), 19.5, 18.0, 16.1 (br), -1.58 (3 C); IR (neat) 3423, 2923, 1575, 1466; HRMS (DEI) Calcd for $\mathrm{C}_{29} \mathrm{H}_{60} \mathrm{NO}_{4} \mathrm{Si}\left(\mathrm{MH}^{+}\right)$514.4292, found 514.4295.

2,2,2-Trichloroethyl $(2 \alpha, 3 \alpha, 6 \alpha)$-2-Methyl-3-[[2-(trimethylsilyl)ethoxy]methoxy]-6-[12[(tetrahydro-2H-pyran-2-yl)oxy]dodecyl]-1-piperidinecarboxylate (13). TrocCl (3.5 mL, $25.6 \mathrm{mmol})$ was added to a solution of crude $12(2.6 \mathrm{~g}, 5.1 \mathrm{mmol})$ in $20: 1 \mathrm{CH}_{2} \mathrm{Cl}_{2} /$ pyridine (182 $\mathrm{mL}$ ) at $0{ }^{\circ} \mathrm{C}$. The solution was stirred at $25^{\circ} \mathrm{C}$ for $24 \mathrm{~h}$. The yellow solution was quenched with $\mathrm{H}_{2} \mathrm{O}(150 \mathrm{~mL})$ and extracted with $\mathrm{CH}_{2} \mathrm{Cl}_{2}(3 \times 100 \mathrm{~mL})$. The combined organic layers were washed with 5\% aqueous $\mathrm{NaHCO}_{3}(200 \mathrm{~mL}), 1 \mathrm{M} \mathrm{HCl}(200 \mathrm{~mL})$, dried over $\mathrm{MgSO}_{4}$, and concentrated to give a yellow oil. Flash chromatography on silica gel (99:1) $\mathrm{CHCl}_{3} / \mathrm{MeOH}$ yielded $3.4 \mathrm{~g}(97 \%)$ of 13 as a clear oil: ${ }^{1} \mathrm{H}$ NMR $\left(\mathrm{CDCl}_{3}\right)$ 4.90-4.81 (m, 1), 4.68 (br, 2), 4.594.51 (m, 3), $4.12-4.04$ (br, 1), 3.86-3.80 (m, 1), 3.72-3.34 (m, 4), 3.50-3.43 (m, 1), 3.38-3.31 (m, 1), 1.84-1.14 (m, 35), $0.91(\mathrm{t}, 2, J=8.5),-0.02(\mathrm{~s}, 9) ;{ }^{13} \mathrm{C} \mathrm{NMR}\left(\mathrm{CDCl}_{3}\right)$ 154.0, 98.8, $95.7(\mathrm{~m})$, $93.1(\mathrm{~m}), 75.0(\mathrm{~m}), 74.2(\mathrm{~m}), 67.6,65.3,62.3,50.8,49.8,34.9(\mathrm{~m}), 30.7,29.7-29.4$ (8 C), 27.6 (m), 26.20, 26.15, 25.5, 20.5, 19.7, 18.0, 14.1 (m), -1.5 (3 C); IR (neat) 2924, 1710; HRMS (FAB, $\mathrm{CH}_{2} \mathrm{Cl}_{2} / \mathrm{CHCA}$ ) Calcd for $\mathrm{C}_{32} \mathrm{H}_{60} \mathrm{NO}_{6} \mathrm{NaSiCl}_{3}\left(\mathrm{MNa}^{+}\right)$710.3148, found 710.3116 .

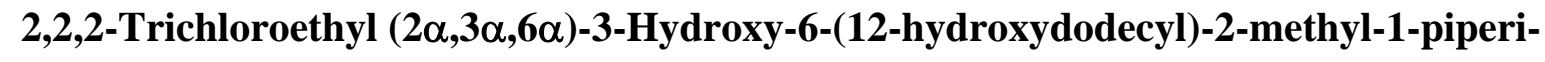
dinecarboxylate (14). 2 M Sulfuric acid (13.6 mL) was added to a solution of 13 (3.5 g, 5.1 $\mathrm{mmol}$ ) in $41 \mathrm{~mL}$ of $\mathrm{MeOH}$. The mixture was heated at $65^{\circ} \mathrm{C}$ for $24 \mathrm{~h}$ and concentrated under reduced pressure. The aqueous layer was basified with $2 \mathrm{M} \mathrm{KHCO}_{3}(40 \mathrm{~mL})$ and extracted with EtOAc $(3 \times 40 \mathrm{~mL})$. The combined organic layers were dried over $\mathrm{Na}_{2} \mathrm{SO}_{4}$ and concentrated to give $2.3 \mathrm{~g}(97 \%)$ of $\mathbf{1 4}$ as a clear oil that was used without purification: ${ }^{1} \mathrm{H} \mathrm{NMR}\left(\mathrm{CDCl}_{3}\right) 4.92-$ $4.59(\mathrm{~m}, 2), 4.51(\mathrm{dq}, 1, J=6.7,6.7), 4.14-4.11(\mathrm{~m}, 1), 3.83-3.79(\mathrm{~m}, 1), 3.64(\mathrm{t}, 2, J=6.4), 1.80$ - 
1.21 (m, 29); ${ }^{13} \mathrm{C} \mathrm{NMR}\left(\mathrm{CDCl}_{3}\right)$ 154.1, 95.7, 75.0, 69.5, 63.0, 51.3, 50.6, 34.8 (br), 32.7, 29.529.4 (7 C), 27.5 (br), 26.1, 25.7, 22.7 (br), 13.5 (m); IR (neat) 3391, 1698; HRMS (DEI) Calcd for $\mathrm{C}_{21} \mathrm{H}_{39} \mathrm{NO}_{4} \mathrm{Cl}_{3}\left(\mathrm{MH}^{+}\right)$474.1945, found 474.1926.

\section{2,2,2-Trichloroethyl (2 $\alpha, 3 \alpha, 6 \alpha)-3-H y d r o x y-2-m e t h y l-6-(12-o x o d o d e c y l)-2-m e t h y l-1-$} piperidinecarboxylate (15). To a solution of $14(311 \mathrm{mg}, 0.65 \mathrm{mmol})$ in $6.6 \mathrm{~mL}$ of $\mathrm{CH}_{2} \mathrm{Cl}_{2}$ at

$0{ }^{\circ} \mathrm{C}$ was added $0.5 \mathrm{M}$ aqueous $\mathrm{KBr}$ solution $(0.65 \mathrm{~mL})$ and TEMPO (5 mg, $\left.0.03 \mathrm{mmol}\right)$. A 0.35 $\mathrm{M}$ aqueous solution of sodium hypochlorite $(2.64 \mathrm{~mL}, 0.92 \mathrm{mmol})$ was slowly added. The orange solution was stirred for $3 \mathrm{~h}$. The aqueous phase was separated and extracted with $\mathrm{CH}_{2} \mathrm{Cl}_{2}$ $(3 \times 10 \mathrm{~mL})$. The combined organic layers were washed with brine, dried over $\mathrm{Na}_{2} \mathrm{SO}_{4}$, and concentrated to give $280 \mathrm{mg}$ of an orange oil. Flash chromatography on silica gel $(24: 1)$ $\mathrm{CHCl}_{3} / \mathrm{MeOH}$ yielded $224 \mathrm{mg}$ (72\%) of 15 as a clear oil followed by $56 \mathrm{mg}$ (18\%) of recovered 14: ${ }^{1} \mathrm{H} \mathrm{NMR}\left(\mathrm{CDCl}_{3}\right) 9.77(\mathrm{t}, 1, J=1.8), 4.90-4.60(\mathrm{br}, 2), 4.52$ (dq, $\left.1, J=6.7,6.7\right), 4.14-4.11$ $(\mathrm{m}, 1), 3.84-3.78(\mathrm{~m}, 1), 2.42(\mathrm{dt}, 2, J=1.8,7.3), 1.77-1.21(\mathrm{~m}, 27)$.

6-(12-Hydroxydodecyl)-2-methyl-3-pyridinol. $2 \mathrm{M}$ Sulfuric acid (0.53 mL) was added to a solution of $11(100 \mathrm{mg}, 0.2 \mathrm{mmol})$ in $1.6 \mathrm{~mL}$ of $\mathrm{MeOH}$. The solution was heated at $65{ }^{\circ} \mathrm{C}$ for $14 \mathrm{~h}$. The solution was cooled to $25^{\circ} \mathrm{C}$ and basified with $2 \mathrm{M} \mathrm{KHCO}_{3}$. The aqueous phase was extracted with EtOAc $(3 \times 5 \mathrm{~mL})$. The combined organic layers were dried over $\mathrm{Na}_{2} \mathrm{SO}_{4}$ and concentrated to give $58 \mathrm{mg}\left(100 \%\right.$ of $95 \%$ pure) of pyridinol as an oil: ${ }^{1} \mathrm{H} \mathrm{NMR}\left(\mathrm{CDCl}_{3}\right) 6.96$ $(\mathrm{d}, 1, J=8.2), 6.85(\mathrm{~d}, 1, J=6.7), 3.63(\mathrm{t}, 2, J=6.7), 2.66(\mathrm{dd}, 2, J=7.9,7.9), 2.46(\mathrm{~s}, 3), 1.65-$ $1.50(\mathrm{~m}, 4), 1.35-1.22(\mathrm{~m}, 16)$.

(2 $\alpha, 5 \alpha, 6 \alpha)$-5-Hydroxy-6-methyl-2-piperidinedodecanol. $5 \% \mathrm{Rh} / \mathrm{Al}_{2} \mathrm{O}_{3}(61 \mathrm{mg})$ was added to a solution of the above pyridinol $(60 \mathrm{mg}, 0.2 \mathrm{mmol})$ in $\mathrm{CH}_{3} \mathrm{OH}(2 \mathrm{~mL})$. The solution was shaken under $50 \mathrm{psi}$ of $\mathrm{H}_{2}$ for $24 \mathrm{~h}$, filtered through Celite, and concentrated to give $58 \mathrm{mg}$ (95\%) of pure $(2 \alpha, 5 \alpha, 6 \alpha)-5$-hydroxy-6-methyl-2-piperidinedodecanol as a white solid: mp 84$86{ }^{\circ} \mathrm{C} ;{ }^{1} \mathrm{H}$ NMR $\left(\mathrm{CD}_{3} \mathrm{OD}\right) 3.63$ (br s, 1), 3.54 (t, 2, $J=6.7$ ), 2.85 (dq, $1, J=1.2,6.7$ ), 2.68-2.61 (m, 1), 1.94-1.90 (m, 1), 1.71-1.28 (m, 25), $1.15(\mathrm{~d}, 3, J=6.7) ;{ }^{13} \mathrm{C}$ NMR $\left(\mathrm{CD}_{3} \mathrm{OD}\right)$ 68.3, 63.2, 57.8, 56.3, 37.8, 33.8, 32.9, 31.1-30.8 (7 C), 27.1, 27.0, 26.4, 18.6; IR (KBr) 3490, 2918, 2850; 
HRMS (DEI) Calcd for $\mathrm{C}_{18} \mathrm{H}_{38} \mathrm{NO}_{2}\left(\mathrm{MH}^{+}\right)$300.2903, found 300.2903. Although the literature data for the optically active natural product are reported in $\mathrm{CDCl}_{3},{ }^{3 \mathrm{c}}$ the synthetic racemic compound is insoluble in $\mathrm{CDCl}_{3}$.

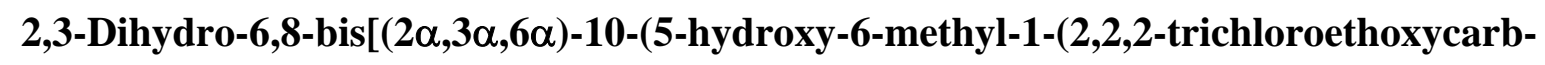
onyl)-2-piperidinyl)decyl]-1H-indolizinium Chloride (16). A solution of 15 (144 mg, 0.3 $\mathrm{mmol}$ ) and 1-pyrroline (5) ${ }^{7}(11 \mathrm{mg}, 0.15 \mathrm{mmol})$ in $1.5 \mathrm{~mL}$ of acetic acid was stirred at $25^{\circ} \mathrm{C}$ for $24 \mathrm{~h}$. The yellow solution was basified with $1 \mathrm{M} \mathrm{NaOH}$. The aqueous layer was washed with $\mathrm{Et}_{2} \mathrm{O}(2 \times 5 \mathrm{~mL})$, saturated with $\mathrm{NaCl}$, and extracted with $\mathrm{CHCl}_{3}(3 \times 50 \mathrm{~mL})$. The combined $\mathrm{CHCl}_{3}$ layers were dried over $\mathrm{CaCl}_{2}$ and concentrated to provide an orange oil. Flash chromatography on silica gel $\left(4: 1 \mathrm{CHCl}_{3} / \mathrm{MeOH}\right)$ followed by filtration through ion exchange resin yielded $75 \mathrm{mg}$ (49\%) of pure 16 as a pale yellow oil: ${ }^{1} \mathrm{H} \mathrm{NMR}\left(\mathrm{CDCl}_{3}\right) 9.47(\mathrm{~s}, 1), 7.83(\mathrm{~s}$, 1), 5.39-5.34 (m, 2), 4.96-4.78 (m, 2), 4.78-4.58 (m, 2), 4.53-4.48 (m, 2), 4.24-4.21 (m, 2), 3.85$3.81(\mathrm{~m}, 2), 3.42$ (t, 2, $J=7.9), 2.81$ (t, 2, $J=7.9), 2.72$ (t, 2, $J=7.9), 2.58$ (tt, 2, $J=7.9,7.9$ ), 1.74-1.19 (m, 50); ${ }^{1} \mathrm{H}$ NMR $\left(\mathrm{CD}_{3} \mathrm{OD}\right) 8.61(\mathrm{~s}, 1), 8.17$ (s, 1), 4.98-4.90 (m, 2), 4.84 (t, 2, J= 7.6), 4.78-4.68 (m, 2), 4.54-4.40 (m, 2), 4.18-4.11 (m, 2), 3.76-3.68 (m, 2), 3.47 (t, 2, J=7.3), $2.81(\mathrm{t}, 4, J=7.6), 2.51(\mathrm{tt}, 2, J=7.6,7.6), 1.80-1.15(\mathrm{~m}, 50) ;{ }^{13} \mathrm{C} \mathrm{NMR}\left(\mathrm{CD}_{3} \mathrm{OD}\right) 156.6,155.7$ (2 C), 146.0, 142.9, 140.7, 139.0, 97.4 (2 C), 76.2 (2 C), 70.3 (2 C), 60.7, 53.0 (2 C), 52.3 (2 C), 36.1 (br, 2 C), [33.3, 32.9, 31.9, 31.8, and 30.8-30.2 (17 C)], 28.9 (br, 2 C), 27.4 (2 C), 23.4 (2 C), 22.2, 14.2 (br, 2 C); IR (neat) 3320, 2927, 2854, 1704; HRMS (FAB, $\mathrm{CH}_{2} \mathrm{Cl}_{2} / \mathrm{CHCA}$ ) Calcd for $\mathrm{C}_{46} \mathrm{H}_{74} \mathrm{~N}_{3} \mathrm{O}_{6} \mathrm{Cl}_{6}\left(\mathrm{M}^{+}\right)$974.3703, found 974.3709.

2,3-Dihydro-6,8-bis[(2 $\alpha, 3 \alpha, 6 \alpha)-10-(5-h y d r o x y-6-m e t h y l-2-p i p e r i d i n y l) d e c y l]-1 H$ indolizinium Chloride Bis Hydrochloride Salt (2•(HCl $\left.)_{2}\right)$ and 2,3-Dihydro-6,8bis[(2 $\alpha, 3 \alpha, 6 \alpha)-10-(5-h y d r o x y-6-m e t h y l-2-p i p e r i d i n y l) d e c y l]-1 H$-indolizinium Chloride (Juliprosine, 2). Zn dust (174 mg, $2.7 \mathrm{mmol})$ was added to a solution of 16 (90 mg, $0.09 \mathrm{mmol})$ in $1.0 \mathrm{~mL}$ of $\mathrm{MeOH}$ and $10 \mu \mathrm{L}$ of conc $\mathrm{HCl}$. The solution was heated at $65^{\circ} \mathrm{C}$ for $3 \mathrm{~h}$, cooled, filtered through Celite, and concentrated to give a pale yellow oil. Flash chromatography on silica gel $(99: 1 \mathrm{MeOH} / \mathrm{HCl})$ followed by filtration through ion exchange resin yielded $\mathbf{2} \bullet(\mathbf{H C l})_{2}$ 
as a pale yellow oil: ${ }^{1} \mathrm{H}$ NMR $\left(\mathrm{CD}_{3} \mathrm{OD}\right) 8.63(\mathrm{~s}, 1), 8.17(\mathrm{~s}, 1), 4.85(\mathrm{t}, 2, J=7.6), 3.84$ (br s, 2), $3.48(\mathrm{t}, 2, J=7.6), 3.25(\mathrm{dt}, 2, J=6.7,6.7), 3.19-3.05(\mathrm{~m}, 2), 2.80(\mathrm{t}, 4, J=6.7), 2.51(\mathrm{tt}, 2, J=$ 7.6, 7.6), 1.96-1.91 (m, 2), 1.82-1.32 (m, 48); ${ }^{13} \mathrm{C}$ NMR ( $\left.\mathrm{CD}_{3} \mathrm{OD}\right)$ 156.7, 146.0, 143.0, 140.9, 139.0, 66.0 (2 C), 60.7, 58.8 (2 C), 57.7 (2 C), 34.9 (2 C), [33.3, 32.9, 32.0, 31.8, 31.1, and 30.830.4 (19 C)], 26.5 (2 C), 23.7 (2 C), 22.2, 16.1 (2 C). These data are identical to those of an authentic sample of the trication $\mathbf{2} \cdot(\mathbf{H C l})_{2}$ kindly provided by Prof. Manfred Hesse.

The trication $\mathbf{2} \cdot(\mathbf{H C l})_{2}$ was taken up in $\mathrm{CHCl}_{3}(5 \mathrm{~mL})$ and $6 \mathrm{M}$ aqueous $\mathrm{NH}_{3}(5 \mathrm{~mL})$. The mixture was shaken and the organic layer separated. The aqueous phase was extracted with $\mathrm{CHCl}_{3}(3 \times 5 \mathrm{~mL})$. The combined organic layers were dried over $\mathrm{CaCl}_{2}$ and concentrated to yield $42 \mathrm{mg}(72 \%)$ of 2 as a clear oil: ${ }^{1} \mathrm{H}$ NMR $\left(\mathrm{CDCl}_{3}\right) 9.40(\mathrm{~s}, 1), 7.82(\mathrm{~s}, 1), 5.33(\mathrm{t}, 2, J=$ 7.6), 3.54 (br s, 2), 3.41 (t, 2, $J=7.6), 2.81(\mathrm{dd}, 2, J=7.9,7.9), 2.79-2.72(\mathrm{~m}, 2), 2.71(\mathrm{dd}, 2, J=$ 7.9, 7.9), $2.57(\mathrm{tt}, 2, J=7.9,7.9), 2.56-2.50(\mathrm{~m}, 2), 1.92-1.87(\mathrm{~m}, 2), 1.66-1.23(\mathrm{~m}, 42), 1.09(\mathrm{~d}$, 6, $J=6.7) ;{ }^{13} \mathrm{C} \mathrm{NMR}\left(\mathrm{CDCl}_{3}\right)$ 153.5, 143.8, 141.9, 139.6, 138.6, 67.9 (2 C), 59.8, 57.14, 57.12, 55.74, 55.71, 36.9, 36.8, [32.5, 32.04, 32.01, 30.64, 30.59, and 29.8-29.1 (19 C)], 26.03, 25.96, 25.73, 25.71, 21.4, 18.7, 18.6; IR (neat) 3319, 2925, 2853, 1695; UV (EtOH) $\lambda_{\max }, \mathrm{nm}(\log \varepsilon)$ : 276 (3.68), 286 (sh, 3.58); HRMS (FAB, MeOH/NBA) Calcd for $\mathrm{C}_{40} \mathrm{H}_{72} \mathrm{~N}_{3} \mathrm{O}_{2}\left(\mathrm{M}^{+}\right) 626.5625$, found 626.5617. These data are identical to those reported for the natural product. $^{2}$

trans-6,8-Diheptyl-1,2,3,5,8,8a-hexahydro-6,8-indolizine (18t) and cis-6,8-Diheptyl1,2,3,5,8,8a-hexahydroindolizine (18c). $\mathrm{NaBH}_{4}(210 \mathrm{mg}, 5.6 \mathrm{mmol})$ was added to a solution of 3a $(255 \mathrm{mg}, 0.8 \mathrm{mmol})$ in $1.6 \mathrm{~mL}$ of EtOH. The solution was stirred at $25^{\circ} \mathrm{C}$ for $30 \mathrm{~min}$ and refluxed for $30 \mathrm{~min}$. The solution was then cooled to $25^{\circ} \mathrm{C}$ and poured into a $10 \% \mathrm{NaOH}$ solution. The aqueous phase was extracted with $\mathrm{CHCl}_{3}(3 \times 25 \mathrm{~mL})$, dried over $\mathrm{Na}_{2} \mathrm{SO}_{4}$, filtered and concentrated to give $175 \mathrm{mg}(88 \%)$ of a 1:1 mixture of cis and trans isomers as a yellow oil. Flash chromatography on silica gel (97:3:0.05 $\mathrm{CHCl}_{3} / \mathrm{MeOH} / 6 \mathrm{M}$ aqueous $\left.\mathrm{NH}_{3}\right)^{26}$ yielded $84 \mathrm{mg}$ (42\%) of 18t followed by $84 \mathrm{mg}(42 \%)$ of $18 c$.

Data for (18t): ${ }^{1} \mathrm{H} \mathrm{NMR}\left(\mathrm{CDCl}_{3}\right) 5.34(\mathrm{~s}, 1), 3.32(\mathrm{~d}, 1, J=15.5), 3.17$ (br dd, $1, J=8.2$, 8.2), 2.62 (d, 1, $J=15.5), 2.11(\mathrm{ddd}, 1, J=8.2,8.2,8.2), 2.06-1.95$ (m, 4), 1.89-1.80 (m, 1), 
1.80-1.68 (m, 2), 1.50-1.35 (m, 5), 1.35-1.14 (m, 18), $0.883(\mathrm{t}, 3, J=7.3), 0.877(\mathrm{t}, 3, J=7.3)$;

${ }^{13} \mathrm{C} \mathrm{NMR}\left(\mathrm{CDCl}_{3}\right)$ 136.1, 123.9, 65.5, 55.3, 54.6, 42.7, 35.1, 33.2, 31.9, 31.8, 30.0, 29.6, 29.33, 29.27, 29.2, 28.0, 26.6, 22.6 (2 C), 21.4, 14.1 (2 C); IR (neat) 2956, 2925, 2855, 2781, 1460; HRMS (DEI) Calcd for $\mathrm{C}_{22} \mathrm{H}_{41} \mathrm{~N}\left(\mathrm{M}^{+}\right)$319.3239, found 319.3237.

Data for (18c): ${ }^{1} \mathrm{H}$ NMR $\left(\mathrm{CDCl}_{3}\right) 5.54(\mathrm{~d}, 1, J=4.3), 3.33(\mathrm{~d}, 1, J=15.9), 3.18(\mathrm{ddd}, 1, J=$ 8.2, 8.2, 1.5), 2.59 (d, 1, J = 15.9), 2.31-2.25 (m, 1), 2.14-2.04 (m, 2), $1.96(\mathrm{t}, 2, J=7.3), 1.85-$ $1.60(\mathrm{~m}, 4), 1.52-1.35(\mathrm{~m}, 4), 1.32-1.20(\mathrm{~m}, 18), 0.88(\mathrm{t}, 6, J=7.3) ;{ }^{13} \mathrm{C} \mathrm{NMR}\left(\mathrm{CDCl}_{3}\right) 135.7$, $124.2,63.6,56.4,55.1,36.9,35.1,31.92,31.86,30.2,30.1,29.4,29.3,29.2,28.0,27.9,25.2$, 22.7 (2 C), 21.7, 14.1 (2 C); IR (neat) 2925, 2855, 2782, 1460; HRMS (DEI) Calcd for $\mathrm{C}_{22} \mathrm{H}_{41} \mathrm{~N}$ $\left(\mathrm{M}^{+}\right)$319.3239, found 319.3249.

Bis(2,2,2-trichloroethyl) $(2 \alpha, 3 \alpha, 6 \alpha)-6,6$ '-[trans-(1,2,3,5,8,8a-Hexahydro-6,8-indolizinediyl)di-10,1-decanediyl]bis[3-hydroxy-2-methyl-1-piperidinecarboxylate] (24t) and Bis(2,2,2-trichloroethyl) $(2 \alpha, 3 \alpha, 6 \alpha)-6,6$ '-[cis-(1,2,3,5,8,8a-Hexahydro-6,8-indolizinediyl)di10,1-decanediyl]bis[3-hydroxy-2-methyl-1-piperidinecarboxylate] (24c). $\mathrm{NaBH}_{4}(10 \mathrm{mg}$, $0.26 \mathrm{mmol})$ was added to a solution of $16(38 \mathrm{mg}, 0.04 \mathrm{mmol})$ in $1 \mathrm{~mL}$ of EtOH. The solution was stirred at $25{ }^{\circ} \mathrm{C}$ for $30 \mathrm{~min}$ and at reflux for $30 \mathrm{~min}$. The solution was then cooled to $25^{\circ} \mathrm{C}$ and poured into $3 \mathrm{~mL}$ of $10 \%$ aqueous $\mathrm{NaOH}$. The solution was extracted with $\mathrm{CHCl}_{3}(3 \times 5$ $\mathrm{mL}$ ). The combined $\mathrm{CHCl}_{3}$ layers were dried over $\mathrm{Na}_{2} \mathrm{SO}_{4}$ and concentrated to give $28 \mathrm{mg}$ (78\%) of a 1:1 mixture of $\mathbf{2 4 t}$ and $\mathbf{2 4 c}$ as a yellow oil. Flash chromatography on silica gel (97:3:0.05 $\left.\mathrm{CHCl}_{3} / \mathrm{MeOH} / 6 \mathrm{M} \mathrm{NH}_{3}\right)^{26}$ yielded $14 \mathrm{mg}(39 \%)$ of $\mathbf{2 4 t}$ followed by $14 \mathrm{mg}$ (39\%) of 24c.

Data for (24t): ${ }^{1} \mathrm{H}$ NMR $\left(\mathrm{CDCl}_{3}\right) 5.35$ (s, 1), 4.91-4.59 (br, 4), 4.51 (dq, 2, $\left.J=6.7,6.7\right)$, 4.18-4.09 (m, 2), 3.84-3.78 (m, 2), $3.32(\mathrm{~d}, 1, J=15.3), 3.21-3.14(\mathrm{~m}, 1), 2.63(\mathrm{~d}, 1, J=15.3)$, 2.14-1.18 (m, 61).

Data for (24c): ${ }^{1} \mathrm{H}$ NMR $\left(\mathrm{CDCl}_{3}\right) 5.54(\mathrm{~d}, 1, J=4.9), 4.91-4.60$ (br, 4), 4.51 (dq, 2, $J=6.7$, 6.7), 4.18-4.09 (m, 2), 3.84-3.78 (m, 2), $3.32(\mathrm{~d}, 1, J=15.3), 3.21-3.14(\mathrm{~m}, 1), 2.58(\mathrm{~d}, 1, J=$ 15.3), 2.30-1.18 (m, 61). 
(2 $\alpha, 3 \alpha, 6 \alpha)-6,6$ '-[trans-(1,2,3,5,8,8a-Hexahydro-6,8-indolizinediyl)di-10,1-decanediyl]bis[2-methyl-3-piperidinol] (Juliprosopine, 17t). KOH (408 mg, $7.3 \mathrm{mmol}$ ) was added to a solution of 24t (41 mg, $0.04 \mathrm{mmol})$ in $2.9 \mathrm{~mL}$ of 2-propanol and $4 \mathrm{~mL}$ of $\mathrm{H}_{2} \mathrm{O}$. The solution was heated at $100{ }^{\circ} \mathrm{C}$ in a sealed tube for $2 \mathrm{~d}$. The reaction mixture was cooled. $\mathrm{CHCl}_{3}(20 \mathrm{~mL})$ was added and the organic phase separated. The aqueous phase was extracted with $\mathrm{CHCl}_{3}(4 \times 20$ $\mathrm{mL}$ ). The combined organic layers were dried over $\mathrm{Na}_{2} \mathrm{SO}_{4}$, filtered, and concentrated to give 24 mg (94\%) of $17 \mathrm{t}$ as a clear oil: ${ }^{1} \mathrm{H} \mathrm{NMR}\left(\mathrm{CDCl}_{3}\right) 5.34$ (s, 1), 3.54 (br s, 2), $3.32(\mathrm{~d}, 1, J=15.3)$, 3.17 (br dd, 1, $J=8.5,8.5), 2.75$ (br q, 1, $J=6.7), 2.62$ (d, 1, $J=15.3), 2.60-2.52$ (m, 2), 2.11

(ddd, $1, J=8.5,8.5,8.5), 2.14-1.14(\mathrm{~m}, 55), 1.09(\mathrm{~d}, 6, J=6.7) ;{ }^{13} \mathrm{C} \mathrm{NMR}\left(\mathrm{CDCl}_{3}\right) 136.1,123.9$, 68.0 (2 C), 65.5, 57.2 (2 C), 55.8 (2 C), 55.3, 54.6, 42.7, 37.0 (2 C), 35.1, 33.1, 32.1 (2 C), 30.029.4 (13 C), 28.0, 26.6, 26.2 (2 C), 25.8 (2 C), 21.4, 18.7 (2 C); IR (neat) 3283, 2924, 2852, 2732; HRMS (FAB, $\mathrm{CH}_{2} \mathrm{Cl}_{2} / \mathrm{NBA}$ ) Calcd for $\mathrm{C}_{40} \mathrm{H}_{76} \mathrm{~N}_{3} \mathrm{O}_{2}\left(\mathrm{MH}^{+}\right)$630.5938, found 630.5962. These data are identical to those reported for the natural product. ${ }^{3 a}$

(2 $\alpha, 3 \alpha, 6 \alpha)-6,6$ '-[cis-(1,2,3,5,8,8a-Hexahydro-6,8-indolizinediyl)di-10,1decanediyl]bis[2-methyl-3-piperidinol] (17c). KOH (408 mg, $7.3 \mathrm{mmol}$ ) was added to a solution of 24c (39.5 mg, $0.04 \mathrm{mmol})$ in $2.9 \mathrm{~mL}$ of 2-propanol and $4 \mathrm{~mL}$ of $\mathrm{H}_{2} \mathrm{O}$. The solution was heated at $100{ }^{\circ} \mathrm{C}$ in a sealed tube for $2 \mathrm{~d}$. The reaction mixture was cooled. $\mathrm{CHCl}_{3}(20 \mathrm{~mL})$ was added and the organic phase separated. The aqueous phase was extracted with $\mathrm{CHCl}_{3}(4 \times$ $20 \mathrm{~mL}$ ). The combined organic layers were dried over $\mathrm{Na}_{2} \mathrm{SO}_{4}$, filtered, and concentrated to give $24 \mathrm{mg}(94 \%)$ of $17 \mathrm{c}$ as a clear oil: ${ }^{1} \mathrm{H} \mathrm{NMR}\left(\mathrm{CDCl}_{3}\right) 5.53$ (d, 1, J=4.3), 3.54 (br s, 2), 3.33 (d, $1, J=15.9$ ), 3.18 (br dd, 1, $J=8.0,8.0), 2.75$ (br q, 1, $J=6.7), 2.68-2.52$ (m, 2), 2.29-1.26 (m, 59), $1.09(\mathrm{~d}, 6, J=6.7) ;{ }^{13} \mathrm{C} \mathrm{NMR}\left(\mathrm{CDCl}_{3}\right)$ 135.2, 124.2, 68.0 (2 C), 63.5, 57.2 (2 C), 56.4, 55.7 (2 C), 55.1, 37.0 (2 C), 36.9, 35.1, 32.1 (2 C), 30.1-29.3 (13 C), 28.0, 27.9, 26.2 (2 C), 25.8 (2 C), 25.1, 21.7, 18.7 (2 C); IR (neat) 3385, 2924, 2853, 2732.

\section{References}

(26) Reimann, E.; Friesinger, J. M. Arch. Pharm. (Weinheim, Ger.) 1988, 321, 13-16. 